\title{
Realized Volatility Analysis from Various Perspectives Based on Hilbert Huang Transform
}

\author{
Sizhe Hou ${ }^{1}$, Jiangrui Chen ${ }^{2}$, Lianqian Yin ${ }^{3}$, Wei Zhang ${ }^{2}$, Xiaojie Liu ${ }^{1} \&$ Haoting $\mathrm{Li}^{2}$ \\ ${ }^{1}$ School of Electrical and Information, Jinan University, Zhuhai, China \\ ${ }^{2}$ International Business School, Jinan University, Zhuhai, China \\ ${ }^{3}$ Finance Department of International Bussiness School, Jinan University, Zhuhai, China \\ Correspondence: Lianqian Yin, Finance Department of International Bussiness School, Jinan University, \\ Qianshan road 206\#, Zhuhai City, Guangdong Province, Post No 519070, China. Tel: 86-135-7064-5138. E-mail: \\ lianqian@jnu.edu.cn
}

Received: August 31, 2015

Accepted: September 29, $2015 \quad$ Online Published: November 25, 2015

doi:10.5539/ijef.v7n12p189

URL: http://dx.doi.org/10.5539/ijef.v7n12p189

\begin{abstract}
In this paper, based on results of the volatility of stock returns after the Hilbert Huang Transform, to research the influential factors of volatility composition, the influential factor model of yield volatility is established. This model studies the volatility from three angles respectively: the hysteresis of impact, the influence degree and the affect correlation. For the hysteresis of impact, this paper uses the ARMA model to determine lag phases of different $I M F$ of volatility. For the influence degree, after using principal component analysis to eliminate the multicollinearity between different $I M F$, we calculate direct contribution, correlation coefficient and variable coefficient to quantify the influence degree of $I M F$ on $R V, B V$ and $J V$, the independence degree and the information abundance. For affect correlation, this paper adopts four different distance calculating methods and grey correlation method to depict the connection degree between $R V$ and $I M F$ in different dimensions. Finally, this paper uses the data of China's financial markets to carry on the empirical analysis, and explores various characteristics of realized volatility through comprehensive influence degree, in order to provide new perspectives and ideas for financial analysis and forecast.
\end{abstract}

Keywords: the volatility of stock returns, Hilbert-Huang Transform (HHT), the hysteresis of impact, the influence degree, the affect correlation

\section{Introduction}

The fluctuation of stock prices is unpredictable and may change radically because of some factors. So analyzing and forecasting the volatility has the significance of controlling risk and stabilizing the financial markets.

Stock price volatility is defined as the stock returns (stock price change ratio) standard deviation. According to explanation in economic sense, the principal causes of volatility are from the following three aspects: one is the macroeconomic impact on a specific industry sector, known as the system risk; the second one is the specific event impacts on an enterprise, known as unsystematic risk; the third one is effects of changes of investors' psychological states and anticipation on target price. Due to the numerous influencing factors of volatility and complex relation between each other, so the study of the formation of volatility and influence degree among factors is of great importance to modern economy and financial markets.

As a new kind of non-stationary signal analysis tool, the Hilbert - Huang Transform (HHT) has been widely used in meteorology, biomedicine, structural mechanics, signal processing and etc. Based on the time series of data, the HHT method is appropriate for researches in the field of finance as a kind of detection method with high precision, high robustness and strong adaptability. According to the research experience of previous scholars, using HHT method for the analysis of financial time series data can better identify the sequence of hidden fluctuation cycle. The modeling analysis results by the HHT method of financial time series data is superior to traditional wavelet analysis, kalman filter analysis and other time series analysis methods. After a large number of experiments, the estimated cycle of time sequences has good accuracy. So it can effectively reflect cycle rules and reduce risks of analysis errors when adopting the HHT to predict the financial cycle. Therefore, the introduction of the HHT method will enrich data analysis methods of financial time series, improve and perfect 
the analysis tools of non-stationary time series, hence promote modeling developments in the China's financial markets(Yong Li, 2013).

Huang (2003) has firstly carried out the promotion of the HHT method to financial applications and introduced the direction of potential applications for financial time series analysis. Guhathakurta (2006) has done the stock index prediction using the HHT method. Islam (2012) has accomplished financial time series decomposition using multidimensional EMD methods, and compared the analysis results with the wavelet decomposition, concluding that the EMD decomposition is of better prediction effect. Luan Shibao (2008) has studied the periodicity of stock index with the HHT method, and analyzed the economic significance of IMF components. Teng Fei (2008) has applied the HHT method to analysis of high frequency data of Shanghai and shenzhen 300 index and found the approximated corresponding relations between approximate periodic signal frequency with abundant high frequency content and the periodic. Ding Zhihong (2009) has decomposed the daily yields of csi 300 index using EMD method and argued that the csi 300 index is cyclical in different time scales. Bi Xing (2010) has applied the EMD method to the de-noising research of stock index, and the results have shown that the EMD decomposition can effectively improve the signal-to-noise ratio of the stock index data.

Based on Hilbert Huang transform, many scholars have explored the economic sense of IMF, the period of volatility in different time scales, and the de-noising and prediction of stock index. From the angle of characteristic analysis, according to the equation of realized jump volatility: $R V=B V+J V$, this article studies comprehensive influence relations among $B V, J V$ and $R V$ under different frequencies.

\section{Modeling}

In this paper, we adopt the HHT method to analyze the volatility of stock index.

First, the volatility of stock price $R V$ is decomposed into the volatility of a continuous sample path $\mathrm{BV}$ and the jumping volatility $J V$. According to the definition of Eric, Poon, and Rockinger $(2004,2006)$, the volatility of stock price is calculated as follows:

$$
\begin{gathered}
R V=\sum_{j=1}^{I=1 / \Delta}\left(p_{j \Delta}-p_{(j-1) \Delta}\right)^{2} \\
B V=(\sqrt{2 / \pi})^{-2} \sum_{j=1}^{I=1 / \Delta}\left|p_{j \Delta}-p_{(j-1) \Delta} \| p_{(j+1) \Delta}-p_{j \Delta}\right| \\
J V=R V-B V
\end{gathered}
$$

Where, $p_{j \Delta}$ is the price for the stock at the moment $j$.

Then the volatility of stock return is decomposed by empirical mode decomposition (EMD) and we obtain IMF functions in different characteristic time scales. Next, IMF is transformed by the HHT method. The transformation process is as follows:

For real time signal $X(t)$ which meets the specific conditions, the Hilbert transform can be described as follows:

$$
\hat{X}(t)=\frac{1}{\pi} \int_{-\infty}^{+\infty} \frac{x(\tau)}{t-\tau} d \tau=X(t) * h(t)
$$

Where, $\hat{X}(t)$ is the sequence after the transformation, $*$ is the linear convolution, $h(t)=\frac{1}{\pi t}$ is defined as the

Hilbert transformer, also known as the Hilbert filter. The positive and negative frequency components of the real signal $X(t)$ have -90 and +90 phase shift respectively after the Hilbert filter, and signal spectrum amplitude does not change.

A complex conjugate is formed with $X(t)$ and $\hat{X}(t)$ as the real part and imaginary part respectively, so as to reconstruct analytical signal:

$$
Z(t)=X(t)+i \hat{X}(t)=a(t) e^{i \theta(t)}
$$

In the formula above, the instantaneous amplitude and instantaneous phase are as follows: 


$$
\begin{gathered}
a(t)=\sqrt{X(t)^{2}+\hat{X}(t)^{2}} \\
\tan \theta(t)=\frac{\hat{X}(t)}{X(t)}
\end{gathered}
$$

Instantaneous frequency $\omega(t)$ is defined as the derivative of instantaneous phase $\theta(t)$ :

$$
\omega(t)=\frac{d \theta(t)}{d t}
$$

The analytic signal corresponding to the original signal can be constructed using the Hilbert transform, which can further extract signal's instantaneous amplitude, instantaneous phase and instantaneous frequency and other characteristic parameters. And it's very important to describe the signal, especially the stationary signal.

\subsection{Feature Analysis of the Implemented Jumping Volatility}

This paper decomposes the solved $R V, B V, J V$ using the Hilbert Huang transform, then studies the comprehensive effect of BV's and JV's $I M F$ in different frequencies on the RV, including the hysteresis quality, influence degree and affect correlation.

Firstly, the yield volatility is decomposed using the Hilbert Huang transform and the stationarity test of IMF is accomplished using the method of unit root. After the stationarity test, by using ARMA model, we calculate the lag phase of different IMF and study the hysteresis effects of different IMF on volatilities in order to infer the response sensitivity. Then, in order to eliminate the multicollinearity of different $I M F$, we use principal component analysis (PCA). After that, we use objective evaluation method and calculate the correlation coefficient and variation coefficient to study the influence degree, the independence degree and the information content. Finally we use similarity distance method and the grey correlation method to study the correlation among $I M F$ of $\mathrm{BV}$ and $\mathrm{JV}$ in different frequencies and $\mathrm{RV}$.

\subsubsection{Analysis on Hysteresis}

Based on the $\operatorname{ARMA}(p, q)$ model, this paper inspects hysteresis impacts of each IMF. Model $\operatorname{ARMA}(p, q)$ (Auto Regressive and Moving Average Model) is an important method for time series study. The model is suitable for analysis in time series which is stable, normal and has the characteristic of zero mean. The expression of $\operatorname{ARMA}(p, q)$ is as follows:

$$
Y_{t}=\beta_{0}+\beta_{1} Y_{t-1}+\beta_{2} Y_{t-2}+\ldots+\beta_{p} Y_{t-p}+\alpha_{0}+\alpha_{1} e_{t-1}+\alpha_{2} e_{t-2}+\ldots+\alpha_{q} e_{t-q}+\mu_{t}
$$

Where, $\alpha_{i}$ and $\beta_{i}$ are coefficients, $e_{i}$ is the error term, $\mu_{t}$ obey the normal distribution of mean as 0 and the variance as 1 .

We obtain the ARMA equations of $R V, B V$, and $J V$ respectively using Matlab, and get the optimal lag phases of regression terms and random disturbance terms based on the minimum criterion AIC.

\subsubsection{Analysis on Impact Degree}

First of all, according to such characteristics as correlation and overlap among different IMF, we adopt PCA (principal component analysis) method to eliminate the multicollinearity of the data. Then, we calculate the direct contribution ratio to analyze the influence degree of different $I M F$ on volatility. Finally, from aspects of independence degree and information content, we study the influence of IMF after dimensionality reduction on the volatility.

\section{(1) Principal component analysis}

There are mainly four basic steps for principal component analysis. First, judge whether the original variables comply with the conditions of the principal component analysis using $K M O$ and Bartlett spherical test.

Then, we standardize the original data in order to eliminate the different orders of magnitude and dimension of the variables, and obtain standardized correlation matrix.

Then, factor variables are constructed. Because practical significance of the factor is not clear, factor rotating is necessary in order to obtain more obvious practical meaning.

Finally, the transformed data are obtained by using a linear combination of the original indexes. 
(2) Direct contribution, correlation coefficient and variance coefficient

Direct contribution method can be utilized to study the influence degree of different IMF. After the decomposition of $I M F$ and PCA, the function of direct contribution is as follows:

$$
\text { Rate }_{i}=\frac{I M F_{i}}{\sum_{i=1}^{n} I M F_{i}}
$$

\section{- Correlation coefficient and variance coefficient}

This paper determines the independence degree and information content of different $I M F$ based on the correlation coefficient and the variation coefficient.

Firstly, correlation coefficient method confirms weights based on the correlation among the indices, that is, the smaller correlation between one index and others, the stronger such index's independence is.

Secondly, variation coefficient method stands for information content. The larger variation from an index is, the more information it contains for the evaluation object.

\subsubsection{Analysis on Correlation}

In the research on the relevance of volatility in the financial market, we need to consider both linear correlation and non-linear correlation at the same time. So this paper uses integrated distance and the grey correlation method to study comprehensive related degree of the internal composition of the volatility.

In this article, we use Manhattan distance, Euclidean distance, Angle cosine, and correlation coefficient to depict integrated distance, so as to get the distance correlation between $J V, R V$ and $B V$.

And grey correlation method is used to depict correlation between $I M F$ resulting from the decomposition of the $J V, B V$ and total volatility $R V$, thereby judging the impact of $B V$ and $J V$ on $R V$ in different time scales.

\section{1) Resemblance Distance Method}

When having correlation research, it is necessary to make similarity measurement by calculating the "Distance" among various samples. In this paper, four types of distance method are adopted to depict the comprehensive resemblance.

Different distance calculation methods have different meanings. First, we use the Minkowski distance, including the Euclidean distance and the Manhattan distance. The Euclidean distance depicts linear distance between the two indices while the Manhattan distance is chessboard distance. In addition, both angle cosine and related distance describe the correlation degree of trend between two variables.

\section{- Minkowski Distance}

The Minkowski distance between two n-dimensional vectors: $a\left(x_{11}, x_{12}, \ldots, x_{1 n}\right)$ and $b\left(x_{21}, x_{22}, \ldots, x_{2 n}\right)$, can be expressed as:

$$
d=\sqrt[p]{\sum_{k=1}^{n}\left|x_{1 k}-x_{2 k}\right|^{p}}
$$

Where, $p$ is a variable parameter. When $p=1$, it is the Manhattan distance. When $p=2$, it is the Euclidean distance. When $p \rightarrow \infty$, it is the Chebyshev distance.

\section{- Angle Cosine}

For two n-dimensional vectors: $a\left(x_{11}, x_{12}, \ldots, x_{1 n}\right)$ and $b\left(x_{21}, x_{22}, \ldots, x_{2 n}\right)$, the concept of cosine can be used to measure their similarity level.

$$
\cos \theta=\frac{\sum_{k=1}^{n} x_{1 k} x_{2 k}}{\sqrt{\sum_{k=1}^{n} x_{1 k}^{2}} \sqrt{\sum_{k=1}^{n} x_{2 k}^{2}}}
$$


The range of angle's cosine is between -1 and 1 , and the bigger it is, the smaller angle between two vectors will be. When the direction of the two vectors overlap, angle cosine reaches maximum 1, when the direction is opposite, it reaches minimum 1.

- Correlation Coefficient and Correlation Distance

$$
\rho_{X Y}=\frac{\operatorname{Cov}(X, Y)}{\sqrt{D(X)} \sqrt{D(Y)}}
$$

Correlation coefficient is a kind of method used to measure correlation degree of random variable $X$ and $Y$, and the range of correlation coefficient is[-1,1]. The greater the absolute value of correlation coefficient shows the higher correlation degree between $X$ and $Y$. When $X$ and $Y$ is in linear correlation, the correlation coefficient value is 1 (positive correlation) or -1 (negative correlation).

\section{2) Grey Correlation Analysis}

Grey correlation analysis is to analyse and determine the influence of system factors through grey correlation degree. In other words, it is a method which is used to measure the contribution of main factors on the system behavior. Correlation degree is the degree of difference between the geometry of the curve. The basic idea is to determine whether close contact based on the geometry of the curve sequence is similar. The closer the curve is, the greater correlation between corresponding sequences is, whereas the less. The greater grey correlation is, the higher consistence degree of two factors' change trend will be.

There is no demand for the size and regularity of samples in grey correlation analysis. It requires small amount of calculation and also doesn't have the condition that qualitative analysis is inconsistent with quantitative results, so we choose it for analysis. The calculation equation is as follows:

$$
\xi_{i j}=\frac{\min _{i} \min _{j}\left\{\Delta_{i j}^{+}\right\}+\rho \max _{i} \max _{j}\left\{\Delta_{i j}^{+}\right\}}{\Delta_{i j}^{+}+\rho \max _{i} \max _{j}\left\{\Delta_{i j}^{+}\right\}}
$$

Where, $\xi_{i j}$ is grey relating modulus; $\Delta_{i j}^{+}$is the distance between the $j$ th index value of the $i$ th object and the optimum value of its index (the distance between $R V$ and $I M F$ of $B V$ and $J V$ in different time scales). And $\rho$ is differentiated coefficient, which can control variation range of grey relating modulus and ranges from 0 to 1. We refer $\rho 0.5$ in this paper.

\section{Empirical Volatility Model in China}

In recent years, with the increasing fluctuation of international financial market, countries around the world face the common issues about how to prevent the volatility risk from financial markets. Firstly, by taking 5 types of stocks from China securities market as the research object, we use the realized volatility signature diagram to determine the optimal sampling frequency as per 5 minutes, which means the time interval from each sample is 5 minutes. Then, we calculate the variance of jumping volatility by the concept of realized volatility. After that, we obtain IMF from different frequencies and trend functions after decomposing the volatility by Hilbert Huang transform. Finally, we make empirical analysis of realized volatility from angles of the duration of influence, the degree of influence and the influence correlation.

\subsection{Selection Rules of Data}

To reflect the comprehensiveness of the study, we select 5 types of security stocks which could reflect China financial market.

From macroscopic aspect, Shanghai Composite Index and Shenzhen Component Index which embody the trend of China financial markets are selected.

From microscopic aspect, Vanke (SZ000002), on the top 200, Dongfeng Motor (SH600006), from 201 to 500, and Yangtze river (SH600119), from 501 to 1000, are selected.

Table 1 . Selection of the security stocks

\begin{tabular}{llllll}
\hline Category & Index & Index & Middle-cap & Small-cap & Small-cap \\
\hline
\end{tabular}




$\begin{array}{llllll}\text { Stock name } & \text { Shanghai Index } & \text { Shenzhen Index } & \text { Vanke } & \text { Dongfeng Motor } & \text { Yangtze river }\end{array}$

For the conveniences, this paper selects frequency of 5 minutes as sampling frequency, known as the second time scales from January 2, 2001 to August 1, 2008

\subsection{Calculation and Decomposition of Volatility}

In order to make deep research on the jump behavior of realized volatility, decomposition on datum is conducted by Hilbert Huang method.
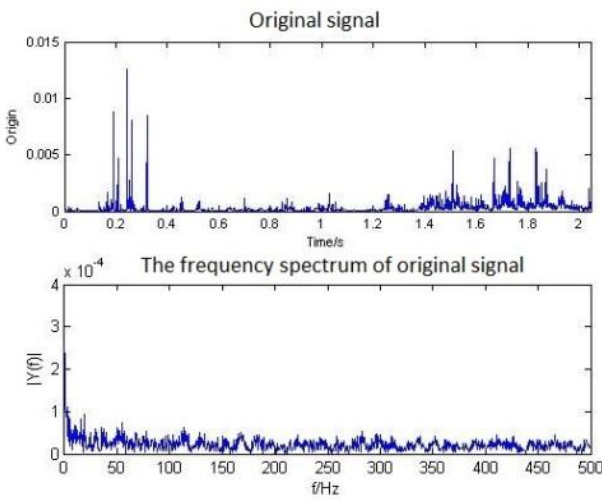

Figure 1. Hilbert Huang decomposition on total volatility $(R V)$ in Shenzhen Component Index
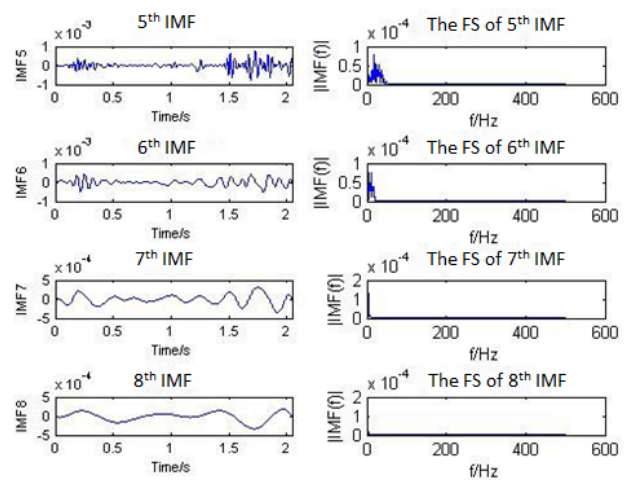

"FS" represents "frequency spectrum"
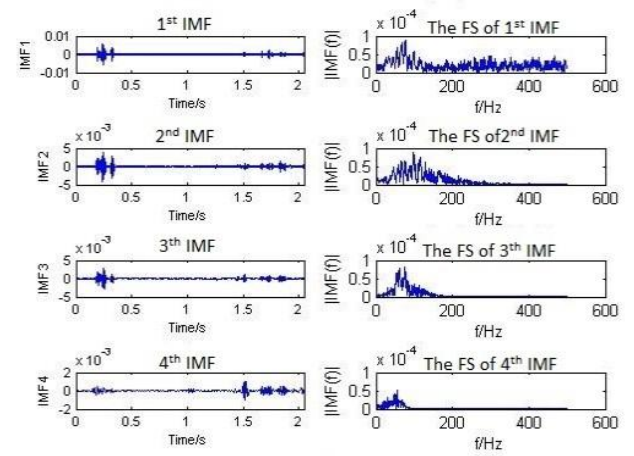

"FS" represents "frequency spectrum"

Figure 2. Hilbert Huang decomposition on total volatility $(R V)$ in Shenzhen Component Index

From Figure 1 the original signals seem perform without any regulation for they are unstable and non-linear figures. From Fig.2, after Hilbert huang decomposition, the total volatility $(R V)$ of Shenzhen Component Index has $11 I M F$ components and a trend term, with frequency from high to low. Similar characteristics could be obtained after decomposition to other volatility involving RV and BV from other types of stock.

\subsection{The Characteristic Analysis Model of Volatility}

Now, we would make empirical analysis of realized volatility in ways of the hysteresis of impact, the degree of influence and the correlation of influence respectively.

\subsubsection{The Hysteresis of Impact-ARMA}

We apply MATLAB software to solve ARMA model equations of $R V, B V$ and $J V$. According to AIC, we get the optimal lag phases of auto-regression terms and stochastic disturbance terms. The result of auto-regression term is as Table 2: 
Table 2. Statistical analysis of hysteresis of RV volatility

\begin{tabular}{cccccccccccc}
\hline IMF & IMF01 & IMF02 & IMF03 & IMF04 & IMF05 & IMF06 & IMF07 & IMF08 & IMF09 & Trend & Average \\
\hline Changjiang & 5 & 5 & 5 & 5 & 5 & 4 & 1 & 1 & 1 & 3 & 3.5 \\
Dongfeng & 4 & 5 & 5 & 5 & 4 & 4 & 4 & 1 & 1 & 1 & 3.4 \\
Shangzheng & 3 & 4 & 4 & 4 & 4 & 4 & 4 & 4 & 4 & & 3.9 \\
Shenzheng & 4 & 4 & 4 & 4 & 4 & 4 & 4 & 4 & 4 & 4 & 3.9 \\
Wanke & 5 & 4 & 5 & 5 & 3 & 5 & 5 & 5 & 5 & 5 & 4.7 \\
Average & 4.2 & 4.4 & 4.6 & 4.6 & 4.0 & 4.2 & 3.6 & 3.0 & 3.0 & 3.3 & 3.9 \\
\hline
\end{tabular}

From the Table 2, we could conclude that the average lag phase of former $I M F$ of $R V$ is bigger, which indicates that the duration of $I M F$ with higher frequency is longer. With the decrease of frequency of each $I M F$, its hysteresis constantly decreases, showing that the $I M F$ of lower frequency enjoys more sensitive reaction from financial market. We can get the same conclusion when analysing the hysteresis of $B V$ and $J V$.

From the perspective of average lag phases of different stocks, the hysteresis of middle-cap stock is the biggest compared to others. Blue chips, known as middle-cap stock, enjoy well business performance, and stable and high cash dividend payment, so their stock prices are greatly influenced by the previous price, with stronger stable state. Small-cap stock refers to the shares of small companies listed on the Shenzhen Component Index. Due to small amount of shares, they react more sensitive to market changes, making the stock price more fluctuant with smaller lag phrase. Component Index reflects overall stock market, so its lag phase is between blue chips and small-cap stocks.

For $B V$, from the perspective of average lag phase of different stocks, it can be seen that three types of stocks share similar lag phases. So we can draw the conclusion that because the $B V$ reflects changes of the stock price after eliminating the jumping movement, it's close to the nature of stock index change rule, which means the lagged effect of early stock prices on later prices for is similar.

The hysteresis of $J V$ has two characteristics: 1) the value of $J V$ in normal circumstances always remains around zero. 2) When an exception occurs, the value of the $J V$ will mutate. At this time, the value of current $J V$ has nothing to do with previous $J V$, so in this case the lag phase is extremely small.

\subsubsection{The Degree of Influence-Principal Component Analysis and Synthesis Weighting Method}

1) Principal component analysis

For the reason that the overlap from each IMF, to some extent, affect the reliability of analysis results, we adopt the principal component analysis to form independent indicators and reduce its dimension in this section, extracting the constituent of eigenvalues whose values are greater than 1 as the main ingredient.

2) Direct empowerment method

Table 3. Weight value of direct empowerment method

\begin{tabular}{ccccccccccc}
\hline Categroy & IMF01 & IMF02 & IMF03 & IMF04 & IMF05 & IMF06 & IMF07 & IMF08 & IMF09 & Trend \\
\hline BV & 0.10 & 0.12 & 0.13 & 0.14 & 0.10 & 0.12 & 0.09 & 0.17 & 0.01 & 0.15 \\
JV & 0.20 & 0.10 & 0.04 & 0.07 & 0.08 & 0.07 & 0.16 & 0.19 & 0.09 & 0.10 \\
RV & 0.21 & 0.14 & 0.06 & 0.12 & 0.08 & 0.09 & 0.12 & 0.14 & 0.03 & 0.12 \\
Average & 0.17 & 0.12 & 0.08 & 0.11 & 0.08 & 0.09 & 0.12 & 0.17 & 0.04 & 0.12 \\
\hline
\end{tabular}

For $R V$, the biggest three weightings are respectively IMF1, IMF2 and IMF8. The weighting of trend term is up to $12 \%$. It is worth noting that the difference between IMF1 and IMF2 is up to 7\%, showing that IMF1 contributes the most to the total volatility.

For $B V$, the biggest three weightings are respectively IMF8, IMF4 and IMF3. The weighting of trend term is up to $15 \%$.

For $J V$, the biggest three weightings are respectively IMF1, IMF8 and IMF7. The weighting of trend term is up to $10 \%$. It is also worth noting that the gaps between the three and other IMF is extraordinarily large, showing that these three $I M F$ contribute to the total volatility the most. 
After comprehensive analysis above, the average weight of IMF1, IMF8 and trend term are extremely big, implying that these three indicators have great influence on the total volatility, making IMF1, IMF8 and trend term important research variables.

3) Variation Coefficient Method and Correlation Coefficient method

By using the variation coefficient method and the correlation coefficient method, this paper quantifies the amount of information contained and independence of $I M F$.

Table 4. Comprehensive empowerment

\begin{tabular}{cccccccccccc}
\hline Category & Weight & IMF01 & IMF02 & IMF03 & IMF04 & IMF05 & IMF06 & IMF07 & IMF08 & IMF09 & Trend \\
\hline \multirow{2}{*}{ BV } & VC. & 0.10 & 0.11 & 0.10 & 0.10 & 0.10 & 0.10 & 0.10 & 0.10 & 0.09 & 0.09 \\
& CC. & 0.05 & 0.04 & 0.05 & 0.07 & 0.08 & 0.08 & 0.12 & 0.13 & 0.21 & 0.19 \\
\multirow{3}{*}{ JV } & VC. & 0.10 & 0.10 & 0.10 & 0.10 & 0.10 & 0.10 & 0.10 & 0.10 & 0.10 & 0.10 \\
& CC. & 0.05 & 0.04 & 0.05 & 0.06 & 0.07 & 0.09 & 0.13 & 0.15 & 0.21 & 0.20 \\
\multirow{2}{*}{ RV } & VC. & 0.10 & 0.10 & 0.10 & 0.10 & 0.10 & 0.10 & 0.10 & 0.10 & 0.10 & 0.10 \\
& CC. & 0.05 & 0.04 & 0.04 & 0.06 & 0.07 & 0.08 & 0.13 & 0.15 & 0.20 & 0.21 \\
\hline
\end{tabular}

Coefficient of variation represents the amount of information. The information weights of different IMF and trend terms in $B V, R V$ and $J V$ are basically the same, about $10 \%$. Based on the above, IMF with different frequencies contain basically the same amount of information.

Correlation coefficient represents the independence of IMF. The coefficients of IMF09 with minimum frequency and trend term are the largest, indicating less relationship between volatility change from these two indicators and change from other IMF. Due to that the trend term reflects the essential rule of the volatility change, its degree of independence is very high, as shown in the Table 4 . With the decrease of the frequency of the $I M F$, the coefficients increase, showing more independence.

Practically, the information from microscopic factors is more abundant and more unstable. Meanwhile, the mutual independence among micro factors is stronger than that among macro factors. For the reason that the macro factors are the results of comprehensive effects of all kinds of micro factors, the fluctuation of macro stocks is less than a single stock, as the index of volatility is less than single stock volatility.

\subsubsection{Analysis on Correlation-Correlation of Distance}

In the research on the relevance of internal composition of financial market volatility, we need to consider the effect of linear correlation and non-linear correlation at the same time. So this paper uses integrated distance method and the grey correlation method to study comprehensive relating degree of the internal composition of volatility.

\section{- Integrated distance method}

This paper uses Manhattan distance, Euclidean distance, Angle cosine, and correlation coefficient to depict integrated distance, so as to get distance correlation between $R V, B V$ and $J V$.

Table 5. Correlation between $B V$ and $R V, J V$ respectively

\begin{tabular}{|c|c|c|c|c|c|c|c|c|}
\hline cjdata & $B V$ & $J V$ & wkdata & $B V$ & $J V$ & dfdata & $B V$ & $J V$ \\
\hline Euclidean distance & 0.151 & 0.849 & Euclidean distance & 0.09 & 0.91 & Euclidean distance & 0.098 & 0.902 \\
\hline Manhattan distance & 0.630 & 0.370 & Manhattan distance & 0.534 & 0.466 & Manhattan distance & 0.640 & 0.360 \\
\hline Angle cosine & 0.981 & 0.019 & Angle cosine & 0.993 & 0.007 & Angle cosine & 0.993 & 0.007 \\
\hline correlation coefficient & 0.989 & 0.011 & correlation coefficient & 0.996 & 0.004 & correlation coefficient & 0.996 & 0.004 \\
\hline Average & 0.688 & 0.312 & Average & 0.653 & 0.347 & Average & 0.682 & 0.318 \\
\hline
\end{tabular}

Table 6.correlation between $B V$ and $R V, J V$ respectively

\begin{tabular}{cccccc}
\hline szdata & $B V$ & $J V$ & shzdata & $B V$ & $J V$ \\
\hline Euclidean distance & 0.497 & 0.503 & Euclidean distance & 0.117 & 0.883 \\
Manhattan distance & 0.807 & 0.193 & Manhattan distance & 0.041 & 0.959 \\
Angle cosine & 0.506 & 0.494 & Angle cosine & 0.988 & 0.012 \\
correlation coefficient & 0.616 & 0.384 & correlation coefficient & 0.982 & 0.018 \\
\hline
\end{tabular}




$\begin{array}{llllll}\text { Average } & 0.607 & 0.394 & \text { Average } & 0.532 & 0.468\end{array}$

Characterization of Euclidean distance is the linear distance between the two indexes. According to data from middle-cap stock and small-cap stock, it can be seen that the connection degree between $B V$ and $R V$ is very small, and the link between $J V$ and $R V$ is great.

Manhattan distance depicts the chessboard distance. From this perspective, the connection degree between $B V$ and $R V$ is greater than it between $J V$ and $R V$. We could get consistent conclusions when using the grey correlation method to depict the connection degree between $I M F$ of $B V, J V$ and $R V$.

The characterization of angle cosine and related distance is the relevance of the trend change between two variables. As shown in the tables above, the change trend of $B V$ and $R V$ is close in middle-cap stock, small-cap stock and market index, and the relating degree is about 0.99 .

Considering all these four distances above, this paper concludes that Yangtze river and Dongfeng Motor, both marked as small-cap stocks, the connection degree between $R V$ and $B V, J V$ respectively shares high similarity, and the connection degree of small-cap stock and mid-cap stock is similar.

\section{- Grey correlation method}

And grey correlation method is used to depict correlation between $I M F$ resulting from the decomposition of the $J V, B V$ and total volatility $R V$, thereby judging the influence of $B V$ and $J V$ on $R V$ in different frequencies. According to the empirical data, in order to make the differences of correlation clearly, we make $\rho 0.1$, and normalize the mutual correlation of IMF with different frequencies (the maximum value is 1 and the minimum value is 0,1$)$. Results are shown in Table 7.

Table 7. Grey correlation analysis of $B V$

\begin{tabular}{cccccccc}
\hline Wave & IMF & SZ & SHZ & WK & DF & CJ & Average \\
\hline BV & IMF01 & 0.863 & 0.819 & 0.988 & 0.909 & 0.825 & 0.88 \\
BV & IMF02 & 0.875 & 0.840 & 0.996 & 0.926 & 0.852 & 0.90 \\
BV & IMF03 & 0.891 & 0.831 & 1.000 & 0.934 & 0.864 & 0.90 \\
BV & IMF04 & 0.902 & 0.826 & 0.990 & 0.952 & 0.854 & 0.90 \\
BV & IMF05 & 0.907 & 0.809 & 0.998 & 0.933 & 0.844 & 0.90 \\
BV & IMF06 & 0.897 & 0.821 & 0.977 & 0.943 & 0.813 & 0.89 \\
BV & IMF07 & 0.901 & 0.820 & 0.999 & 0.952 & 0.658 & 0.87 \\
BV & IMF08 & 1.000 & 0.908 & 0.993 & 0.915 & 0.888 & 0.94 \\
BV & IMF09 & 0.000 & NA & 0.779 & 0.909 & 0.665 & 0.59 \\
BV & IMF10 & 0.238 & NA & NA & NA & NA & 0.24 \\
BV & Trend & 0.764 & 1.000 & 0.910 & 0.802 & 0.842 & 0.86 \\
\hline & & & & & & Average & 0.81 \\
\hline
\end{tabular}

In the Table 7, we can see that the influence of IMF08 resulting from the decomposition of $B V$ has the biggest correlation coefficient with $R V$ from different time scales. We can also see that, BV in frequency of IMF08 has the biggest correlation with $R V$, which is consistent with the above conclusion that IMF08 has the largest direct contribution for $B V$.

Analysis from the perspective of different stock index shows that, $R V$ of the market index is mainly affected by IMF08 of $B V, R V$ of the middle-cap stock is mainly affected by IMF03 of $B V$, and $R V$ of the small-cap stocks are mainly affected by IMF07 and IMF08 of $B V$.

Table 8. Grey correlation analysis of $J V$

\begin{tabular}{lccccccc}
\hline & IMF & SZ & SHZ & WK & DF & CJ & Average \\
\hline JV & IMF01 & 0.779 & 0.852 & 0.414 & 0.361 & 0.285 & 0.54 \\
JV & IMF02 & 0.771 & 0.842 & 0.000 & 0.000 & 0.000 & 0.32 \\
JV & IMF03 & 0.787 & $\mathbf{0 . 8 6 1}$ & 0.260 & 0.212 & 0.093 & 0.44 \\
JV & IMF04 & 0.883 & 0.828 & 0.611 & 0.702 & 0.342 & 0.67 \\
JV & IMF05 & 0.862 & 0.851 & 0.778 & 0.505 & 0.453 & 0.69 \\
JV & IMF06 & 0.849 & 0.854 & 0.811 & 0.501 & 0.586 & 0.72 \\
JV & IMF07 & $\mathbf{0 . 9 0 1}$ & 0.791 & 0.903 & 0.579 & 0.764 & 0.79 \\
JV & IMF08 & 0.787 & 0.213 & $\mathbf{0 . 9 3 0}$ & 0.699 & 0.744 & 0.67 \\
JV & IMF09 & 0.289 & NA & 0.888 & 0.704 & 0.745 & 0.66 \\
JV & IMF10 & 0.633 & NA & NA & NA & NA & 0.63 \\
JV & Trend & 0.852 & 0.000 & 0.437 & $\mathbf{1 . 0 0 0}$ & $\mathbf{1 . 0 0 0}$ & 0.66 \\
\hline
\end{tabular}




\section{Average} 0.62

In the Table 8, we can see that the influence of IMF07 and IMF08 resulting from the decomposition of $J V$ has the biggest correlation coefficient with RV from different time scales. We can also see that, $J V$ in frequency of IMF07 and IMF08 has the biggest correlation with RV, which is consistent with the above conclusion that IMF07 and IMF08 has the largest direct contribution for $B V$.

Analysis from the perspective of different stock index shows that, the trend of $J V$ of the small-cap stocks has the biggest correlation coefficient with $R V$, which indicates that, to some extent, the trend of jump volatility in small-cap stocks is the main influence factor of $R V$. IMF08 of $J V$ in middle-cap stock has the largest correlation coefficient with $R V$.

From a comprehensive view of the Table 7 and Table 8, the comprehensive correlation coefficient between $I M F$ of $B V$ in different time scales and $R V$ is 0.81 , and the comprehensive correlation coefficient between $I M F$ of $J V$ in different time scales and $R V$ is 0.62 . So this paper preliminarily concludes that the influence degree of different $I M F$ of $B V$ on $R V$ is deeper than $I M F$ of $J V$.

\section{Conclusion}

Established by the realized volatility analysis model based on Hilbert Huang transformation, this paper makes an empirical analysis on China financial market. We concentrate on characteristics analysis from three perspective on volatility signals after HHT decomposition. Thus, this article attempts to analyze the characteristics of the financial markets from the angle of their volatilities.

First of all, ARMA model is utilized to study the hysteresis of volatility. Through calculation we could conclude that the lagging effect from latter $I M F$ is less both in large-cap and small-cap volatility so that they respond quickly on volatility of financial market. Therefore, it shows that hysteresis of market fluctuations is derived from the conduction from the latter IMF toward the former IMF, which effects three types of volatility involving $R V, B V$ and $J V$.

Then, we have researched on the independence among the volatility factor, the influence among factor and information abundance of factor through the factor analysis, direct contribution rate, comprehensive empowerment method. The results showed that relative to $J V, B V$ enjoys larger influence for RV, which means that the total volatility is mainly composed of $B V$. On the other hand, $J V$ contains more abundant information compared to $B V$, which means that the total volatility changeable information is mainly determined by $J V$ (Jumping Volatility).

Moreover, considering the concept of 6 kinds of distance to calculate the correlation among $R V, B V$ and $J V$ respectively, the correlation analysis was carried out to analyze the volatility so that we validate the rationality of the equation and the comprehensive influence scope. The comprehensive effects from $B V$ is about $80 \%$ of $R V$, where $J V$ is $20 \%$.

\section{References}

Barndorff-Nielsen, O., \& Shephard, N. (2004). Power and Bipower Variation with Stochastic Volatility and Jumps. Journal of Financial Econometrics, 2(1), 1-37. http://dx.doi.org/10.1093/jjfinec/nbh001

Barndorff-Nielsen, O., \& Shephard, N. (2006). Econometrics of Testing for Jumps in Financial Economics Using Bipower Variation. Journal of Financial Econometrics, 4(1), 1-30. http://dx.doi.org/10.1093/jjfinec/nbi022

Huang, N. E., Wu, M. L., Qu, W. D. et al. (2003). Applications of Hilbert - Huang transform to non-stationary financial time series analysis. Applied Stochastic Models in Business and Industry, 19, 245-268. http://dx.doi.org/10.1002/asmb.501

Huide, L. (2007). Unit Root Test Allowing for Structural Breaks: A Literature Review. The Journal of Quantitative \& Technical Economics, 3, 152-161.

Hui-min, F., \& Cheng-rui, L. (2003). Analysis Method of Correlation Coefficient ARMA(p,q) Series. Journal of Aerospace Power, 2, 161.

Islam, M. R., Al-Mahfuz, M. R., \& Molla, M. K. I. (2012). Multiband Prediction Model for Financial Time Series with Multivariate Empirical Mode Decomposition. Discrete Dynamics in Nature and Society. http://dx.doi.org/10.1155/2012/593018

Kousik, G., Indranil, M., \& Chowdhury, A. R. (2008). Empirical mode decomposition analysis of two different financial time series and their comparison. Chaos, Solitons and Fractals, 37, 1214-1227. http://dx.doi.org/10.1016/j.chaos.2006.10.065 
Shibao, L. (2008). Financial Date Processing Based on Hibert-Huang Transformation. Ocean University of China (Unpublished master dissertation).

Si-feng, L., Hua, C., Ying-jie, Y., \& Ying, C. (2013). Advance in grey incidence analysis modeling. Systems Engineering-Theory \& Practice, 8, 2041-2046.

Tian, L., Jin, T., \& Dai-min, S. (2003). Selection of Sample Length in Unit Root Test. Journal of Applied Statistics and Management, 4, 617-626.

Xing, B., \& Wei, W. (2010). Financial Time Series Analysis Based on EMD and Moving average. Journal of Tianjin University (Social Sciences), 12(2), 125-128.

Yong, L. (2012). Multiresolution Analysis of Financial Times Series-Basing on Hilbert-Huang Transform. University of Science and Technology of China.

\section{Copyrights}

Copyright for this article is retained by the author(s), with first publication rights granted to the journal.

This is an open-access article distributed under the terms and conditions of the Creative Commons Attribution license (http://creativecommons.org/licenses/by/3.0/). 